\title{
Importance of Selecting a Suitable Analysis Frequency Range in Impact Force Identification for Automobile Test Rig
}

\author{
Khoo Shin Yee ${ }^{1, a}$, Ong Zhi Chao ${ }^{1,3, b}$, Kong Keen Kuan ${ }^{1, c}$, Zubaidah Ismail ${ }^{2,3, d}$, \\ Chong Wen Tong ${ }^{1, e}$, Siamak Noroozi ${ }^{4, f}$ and Abdul Ghaffar Abdul Rahman ${ }^{5, g}$
}

\author{
${ }^{1}$ Department of Mechanical Engineering, Engineering Faculty, University of Malaya, 50603 Kuala \\ Lumpur, Malaysia. \\ ${ }^{2}$ Department of Civil Engineering, Engineering Faculty, University of Malaya, 50603 Kuala Lumpur, \\ Malaysia. \\ ${ }^{3}$ Advanced Shock and Vibration Research Group, Applied Vibration Laboratory, Block R, Faculty of \\ Engineering, University of Malaya \\ ${ }^{4}$ School of Design, Engineering \& Computing, Bournemouth University, Poole, Dorset, BH12 5BB, \\ UK \\ ${ }^{5}$ Faculty of Mechanical Engineering, University of Malaysia Pahang, 26600 Pekan, Pahang, \\ Malaysia \\ amikeson.khoo@yahoo.com; akhooshinyee@um.edu.my, balexongzc@um.edu.my,

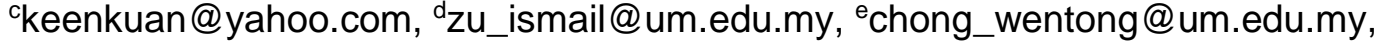 \\ 'snoroozi@bournemouth.ac.uk, 9dragrahman@yahoo.com
}

Keywords: analysis frequency range; data filtering; modal analysis; impact force identification; operating deflection shape analysis; modal transformation method

\begin{abstract}
In this study, the effectiveness of selecting a suitable analysis frequency range in impact force identification is highlighted. A methodology that utilizes Operating Deflection Shape (ODS) analysis, Modal Analysis (MA) and Modal Transformation Method (MTM) to evaluate the dynamic force in three cases of analysis frequency ranges was presented. These three cases are the overestimated, even-estimated, and under-estimated cases, which consist of higher, similar and lower analysis frequency range respectively, compared to the actual excitation frequency range. The performance of this approach was demonstrated via experiment. A Perspex plate with four ground supports was used as the automobile test rig. By measuring the acceleration response and Frequency Response Function (FRF) of the test rig, the time history of unknown force was recovered by the proposed method where the impact location was known in advance. It showed that the force identification result for even-estimated case falls within acceptable range while the force identification result for over-estimated and under-estimated cases is not acceptable.
\end{abstract}

\section{Introduction}

Bump-excited impact force is one of the most common cases, which causes damage to vehicle and reduces the quality of the ride in frequency range of 20 to $100 \mathrm{~Hz}$ [1]. In the case of bumpexcited impact force, direct measurement by using force transducer is not possible due to some difficulties in force sensor configuration. Besides that, installation of a force sensor at the structure's junction, link or support is not feasible because it may alter the boundary condition and dynamic characteristics [2]. Therefore, impact force identification has become one of the key issues in structural design [3].

The information of impact force such as excitation frequency range, impact duration, magnitude, location and line of action is the important database for accurate Computer-Aided Engineering (CAE) simulations. For example, it can be used for trouble-shooting purpose and design 
improvement analysis for noise and vibration [4]. Besides, the force information reflects the structure's health so that action such as structural modification can be taken before material fatigue.

One difficulty faced in impact force identification would be the useful analysis frequency range used to reconstruct the unknown force [5]. The current study initiates an effort to examine the effectiveness of various types of analysis frequency ranges on the impact force identification via Modal Transformation Method (MTM). MTM was used in followed references [6,7]. Assuming the excitation frequency range of impact force is known in advance, the analysis frequency range can be divided into three cases: over-estimated, even-estimated and under-estimated cases. They have analysis frequency ranges which are greater than, equal to and less than the actual excitation frequency range respectively.

\section{Materials and Methods}

Equipment Set-Up. A rectangular shape test rig with 4 ground supports was simulated as vehicle's body and referred as automobile test rig. Fifteen accelerometers were attached on the test rig and numbered as shown in Fig. 1. The rig was used to acquire the responses from 15 discrete locations simultaneously. A modally tuned impact hammer was used to record the time history of impact excitation and it is used for verification purpose. These input and output signals were connected to a laptop through a Data Acquisition (DAQ) system. Meanwhile, post-processing of the data was done using DASYLab ${ }^{\circledR}$, ME'scope $^{\circledR}$ and MATLAB ${ }^{\circledR}$ software.

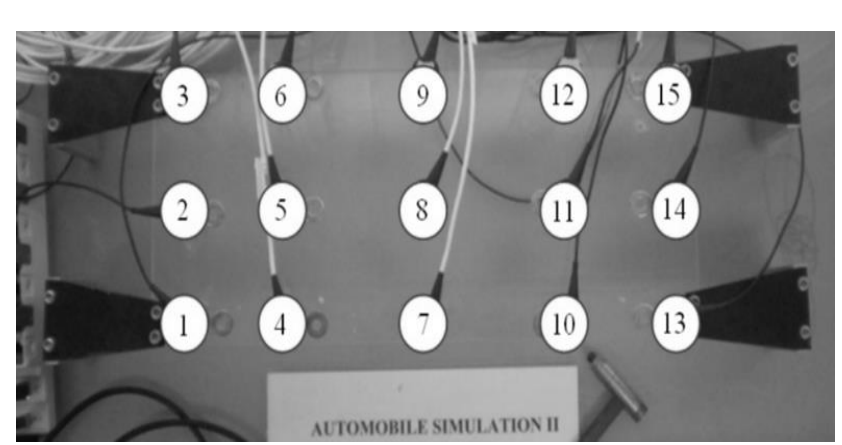

(a)

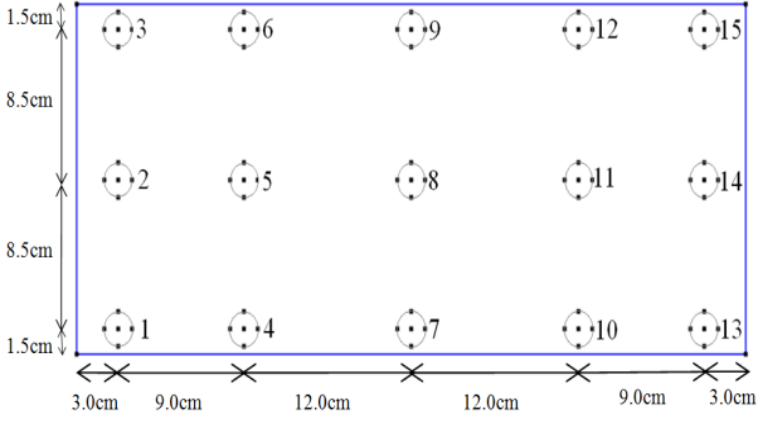

(b)

Fig. 1. Point numbering: (a) vibration test rig, and (b) schematic drawing.

Operating Deflection Shape (ODS). Time-based ODS is used because it is time efficient compared to frequency-based ODS. It helps to record the impact-induced vibrations from 15 accelerometers simultaneously by using a multi-channel DAQ system.

Modal Analysis (MA). Modal analysis is a technique used to determine the inherent dynamic characteristics of a structure which are comprehensively defined by 3 modal parameters (i.e. natural frequencies, mode shapes and damping). Once a single column raw Frequency Response Function (FRF) matrix is measured through FRF measurement [8], these dynamics characteristics are obtained using curve fitting algorithm in ME'Scope ${ }^{\circledR}$ software.

Modal Transformation Method (MTM). Given number of response, mode and force measurements are $n, m$ and $f z$ respectively, MTM is applied to synthesise the FRF using the 3 modal parameters as follows:

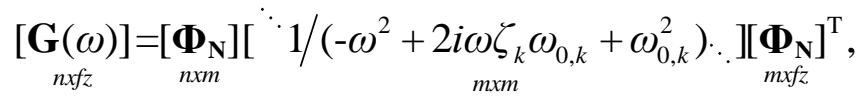


where $[\mathbf{G}(\omega)]$ is a $n$ by $f z$ synthesised FRF matrix. $\left[\boldsymbol{\Phi}_{\mathrm{N}}\right]$ is $n$ by $m$ Unit Modal Mass (UMM) mode shape matrix due to response DOF. $\left[\boldsymbol{\Phi}_{\mathrm{N}}\right]^{\mathrm{T}}$ is $m$ by $f z$ UMM mode shape matrix due to force DOF and it is transpose of $\left[\boldsymbol{\Phi}_{\mathrm{N}}\right] . \omega_{0, k}$ is the $k^{\text {th }}$ mode natural frequency where $k=1,2, \ldots, m . \zeta_{k}$ is the $k^{\text {th }}$ mode damping ratio. $[\because]$ is a diagonal matrix. $\omega$ is angular frequency.

Unknown impact force can be recovered by multiplying pseudo-inverse, pinv of synthesised FRF matrix to raw response vector using Eq. 2. To obtain a least square solution of force identification, it must satisfy $n \geq m \geq f z$.

$$
\{\underset{f \times 1}{Q(\omega)}\}=\underset{n \times f}{\operatorname{pinv}\{[\mathbf{G}(\omega)]\}} \underset{n \times 1}{\not{\boldsymbol{X}}(\omega)}\}
$$

where $\{\ddot{X}(\omega)\}$ is $n$ by 1 acceleration vector. $\{\boldsymbol{Q}(\omega)\}$ is $f z$ by 1 force vector.

According to reference [6], the force identification problem becomes well-posed once impact location is known in advance (i.e. impact location at point 1). Thus the Eq. 2 can be reduced to Eq. 3 as follows:

$$
\left\{Q_{1}(\omega)\right\}=\operatorname{pinv}\left\{\left[\begin{array}{c}
G_{2: 1}(\omega) \\
G_{3: 1}(\omega) \\
\vdots \\
G_{n: 1}(\omega)
\end{array}\right]\right\}\left\{\begin{array}{c}
\ddot{X}_{2}(\omega) \\
\ddot{X}_{3}(\omega) \\
\vdots \\
\ddot{X}_{n}(\omega)
\end{array}\right\}
$$

Impact Force Identification Using Various Types of Analysis Frequency Ranges. In this study, a single unknown impact force acting at point 1 on the test rig was estimated from 15 accelerometers. The excitation frequency range of impact force is known in advance (i.e. 0.5$500.0 \mathrm{~Hz}$ ). The analysis frequency ranges of $\omega$ are selected so that the force identification method can be examined in three cases: over-estimated (i.e. $0.5-999.5 \mathrm{~Hz}$ ), even-estimated (i.e. $0.5-500.0 \mathrm{~Hz}$ ) and under-estimated cases $(0.5-100.1 \mathrm{~Hz})$. Note that the data outside the analysis frequency range is eliminated by setting it to zero and this approach is known as data filtering.

\section{Results and Discussions}

The identified force due to impact at point 1 for over-estimated, even-estimated and underestimated cases are compared with the measured force in time domain as shown in Fig. 2. It was found that the identified force for over-estimated case and under-estimated case did not match the measured force in time domain. The identified force matched the measured force very well for even-estimated case. A lot of large oscillating components were found in over-estimated case compared to the other cases. The correlation coeficient between the measured and identified forces are $0.74,0.91$ and 0.67 for over-estimated, even-estimated and under-estimated cases respectively. Note that correlation coefficient higher than 0.9 indicates a good force identification result and viceversa. Hence, force identification result for even-estimated case falls within acceptable range while the force identification result for over-estimated and under-estimated cases is not acceptable.

The identified force due to impact at point 1 for over-estimated, even-estimated and underestimated cases are compared with the measured force in frequency domain as shown in Fig. 3. Fig. 3(a) shows that the over-estimated case included a significant amount of noise contamination in high frequency region into the force identification. Therefore, this contributes an inaccurate force identification result. Fig. 3(b) shows that the force identification included all the excitation frequency region with negligible noise in high frequency region for even-estimated case. Hence, the 
force identification result is accurate in this case. It is worthwhile to mention that the accuracy of force identification in even-estimated case primarily depends on the accuracy of curve fitting result as discussed in reference [6]. In this case, the correlation between the measured FRF and synthesised FRF is only 0.43 for a frequency range of $0.5-500.0 \mathrm{~Hz}$ while the ideal correlation is above 0.9. So there is still room for improvement by enhancing the curve fitting algorithm. Fig. 3(c) shows that a significant amount of excitation frequency region was not included into the force identification for under-estimated case. In fact, unknown force which is identified through MTM is a summation of mode contribution distributing along the frequency region. There are mode truncation and signal distortion for under-estimated case, therefore the identified force is inaccurate and the magnitude is under-estimated. This explains why its amplitude is less than the amplitude of measured force as shown in Fig. 3(c).

(a) Over-Estimated Case

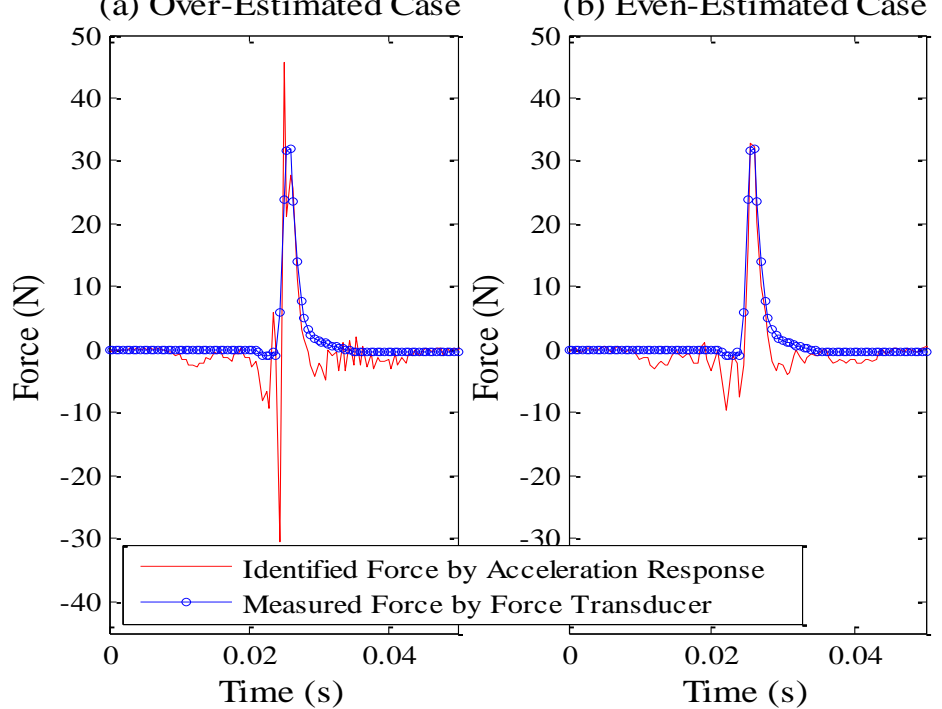

(c) Under-Estimated Case

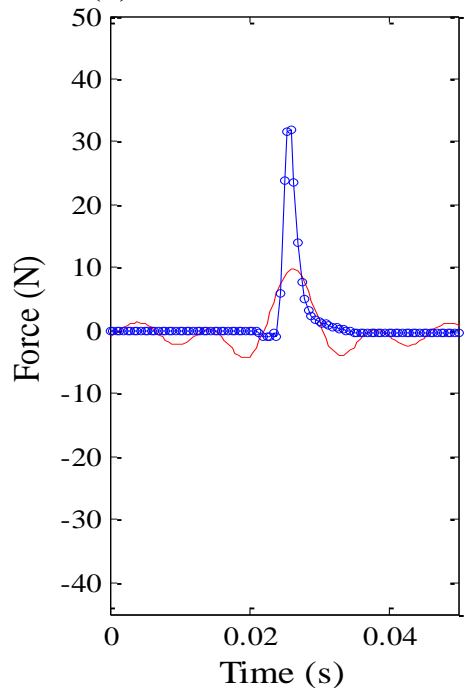

Fig. 2. Comparison between identified force and measured force in time domain for three cases of analysis frequency ranges: (a) over-estimated, (b) even-estimated and (c) under-estimated cases.

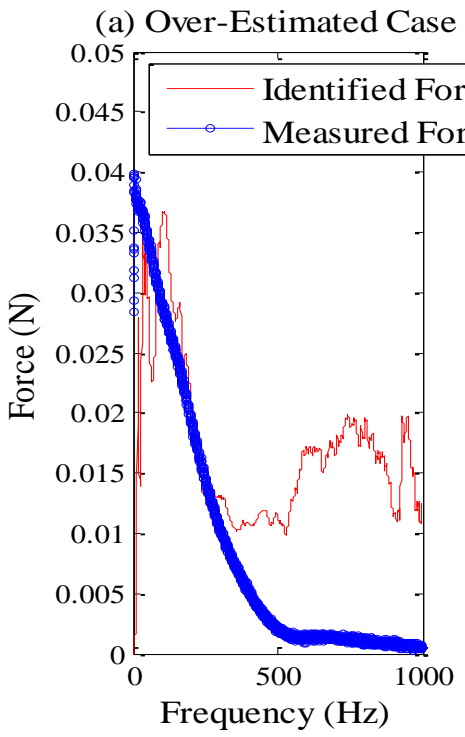

(b) Even-Estimated Case $0.05 \%$

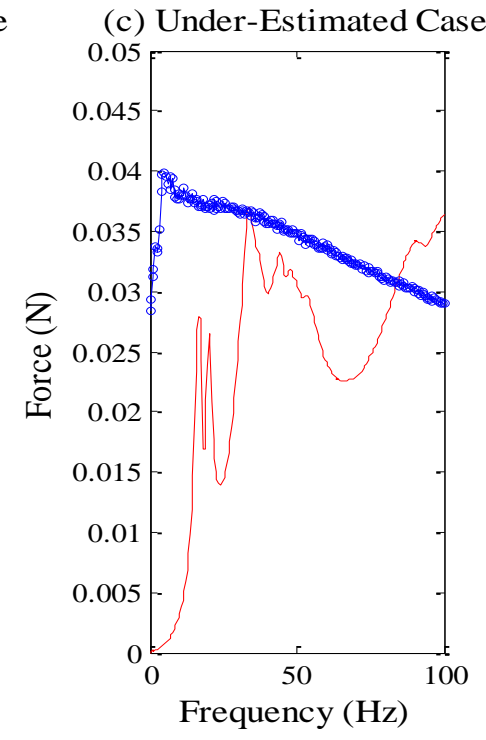

Fig. 3. Comparison between identified force and measured force in frequency domain for three cases of analysis frequency ranges: (a) over-estimated, (b) even-estimated and (c) under-estimated cases.

In general, the analysis frequency range plays an important role in impact force identification. The analysis frequency range shall include the entire excitation frequency region and exclude the 
noise in high frequency region to ensure a reliable and robust force identification result. The current study is conducted for the case of known excitation frequency range. However, impact excitation frequency range is unknown in nature. Signal distortion or high noise contamination will occur if the cut-off frequency is too low or too high. Further research shall be conducted to estimate the analysis frequency range for the case of unknown excitation frequency range.

\section{Summary}

In this study, impact force identification using MTM has been examined in three cases of analysis frequency ranges: over-estimated, even-estimated and under-estimated cases. Results show that even-estimated case which has analysis frequency range equal to the excitation frequency range of impact force has a satisfactory force identification result, while the results for the over-estimated and under-estimated cases are not acceptable. This shows that a suitable analysis frequency range such as the even-estimated case plays an important role in enhancing the result of impact force identification. Further research must be able to extend the current study to estimate the analysis frequency range of impact force for the case of unknown excitation frequency range.

\section{Acknowledgements}

The authors wish to acknowledge the financial support and advice given by Postgraduate Research Fund (PV086-2011A), High Impact Research Grant under UM.C/HIR/MOHE/ENG/15 (D00001516001) and Advanced Shock and Vibration Research (ASVR) Group of University of Malaya.

\section{References}

[1] T. Lee and B. Kim, Vibration analysis of automobile tire due to bump impact, Applied Acoustics. 69 (2008), p. 473-478.

[2] J.-Y. Yoon and R. Singh, Estimation of interfacial forces in a multi-degree of freedom isolation system using a dynamic load sensing mount and quasi-linear models, Journal of Sound and Vibration. 330 (2011), p. 4429-4446.

[3] S.Y. Khoo, Z. Ismail, K.K. Kuan, Z.C. Ong, S. Noroozi, W.T. Chong and A.G.A. Rahman, Impact force identification with pseudo-inverse method on a lightweight structure for underdetermined, even-determined and over-determined cases, International Journal of Impact Engineering, 63 (2014) 52-62. doi: 10.1016/j.ijimpeng.2013.08.005.

[4] T. Otsuka, T. Okada, T. Ikeno, K. Shiomi and M. Okuma, Force identification of an outboard engine by experimental means of linear structural modeling and equivalent force transformation, Journal of Sound and Vibration. 308 (2007), p. 541-547.

[5] A. Cardi, D.E. Adams and S. Walsh. Ceramic body armor single impact force identification on a compliant torso using acceleration response mapping. Structural Health Monitoring. 5 (2006), p. 355-372. doi: 10.1177/1475921706067763.

[6] A.G.A. Rahman, S.Y. Khoo, Z. Ismail, W.T. Chong and S. Noroozi, Impact force identification by using modal transformation method for automobile test rig, in: 4th International Conference on Noise, Vibration and Comfort (NVC), Kuala Lumpur, Malaysia (2012).

[7] A.G.A. Rahman, S.Y. Khoo, Z. Ismail, W.T. Chong and S. Noroozi, Impact force identification by using modal transformation method in collocated and non-collocated cases, in: 2nd International Conference on Mechanical Engineering Research (ICMER), Kuala Lumpur, Malaysia (2013).

[8] W.G., Halvorsen and D.L. Brown, Impulse technique for structural frequency response testing, Sound and Vibration. 11 (1977), p. 8-21. 